\author{
A.K.Y. NGAI ${ }^{1}$ \\ S.T. PERSIJN ${ }^{1}$ \\ I.D. LINDSAY ${ }^{2}$ \\ A.A. KOSTEREV ${ }^{3}$ \\ P. GROß ${ }^{2}$ \\ C.J. LEE ${ }^{2}$ \\ S.M. CRISTESCU ${ }^{1}$ \\ F.K. TITTEL ${ }^{3}$ \\ K.-J. BOLLER ${ }^{2}$ \\ F.J.M. HARREN ${ }^{1,00}$
}

\section{Continuous wave optical parametric oscillator for quartz-enhanced photoacoustic trace gas sensing}

${ }^{1}$ Life Science Trace Gas Facility, Molecular and Laser Physics, Institute for Molecules and Materials, Radboud University, P.O. Box 9010, 6500 GL Nijmegen, Netherlands

${ }^{2}$ Laser Physics and Nonlinear Optics Group, Faculty of Science and Technology, University of Twente, 7500 AE Enschede, Netherlands

${ }^{3}$ Department of Electrical and Computer Engineering, Rice University, Houston, TX 77005, USA

\begin{abstract}
Received: 23 July 2007
Published online: 24 August 2007 • C) Springer-Verlag 2007

ABSTRACT A continuous wave optical parametric oscillator, generating up to $300 \mathrm{~mW}$ idler output in the 3-4 $\mu \mathrm{m}$ wavelength region, and pumped by a fiber-amplified DBR diode laser is used for trace gas detection by means of quartz-enhanced photoacoustic spectroscopy (QEPAS). Mode-hop-free tuning of the OPO output over $5.2 \mathrm{~cm}^{-1}$ and continuous spectral coverage exceeding $16.5 \mathrm{~cm}^{-1}$ were achieved via electronic pump source tuning alone. Online monitoring of the idler wavelength, with feedback to the DBR diode laser, provided an automated closed-loop control allowing arbitrary idler wavelength selection within the pump tuning range and locking of the idler wavelength with a stability of $1.7 \times 10^{-3} \mathrm{~cm}^{-1}$ over at least $30 \mathrm{~min}$.

Using this approach, we locked the idler wavelength at an ethane absorption peak and obtained QEPAS data to verify the linear response of the QEPAS signal at different ethane concentrations $(100 \mathrm{ppbv}-20 \mathrm{ppmv})$ and different power levels. The detection limit for ethane was determined to be $13 \mathrm{ppbv}(20 \mathrm{~s}$ averaging), corresponding to a normalized noise equivalent absorption coefficient of $4.4 \times 10^{-7} \mathrm{~cm}^{-1} \mathrm{~W} / \mathrm{Hz}^{1 / 2}$.
\end{abstract}

PACS 42.55.Wd; 42.65.Yj; 42.62.Fi

\section{Introduction}

The strength of continuous wave, mid-infrared, optical parametric oscillators (OPOs) lies in their ability to cover a broad spectral range, while offering high power and exceptional beam characteristics in terms of beam profile, linewidth and stability [1-6]. This makes the OPO an excellent light source for spectroscopic purposes, especially when it is operated in the $3-5 \mu \mathrm{m}$ wavelength range where many molecules important for life science have strong characteristic absorptions. A highly attractive mode of operation is the use of a singly-resonant OPO where the majority of tuning is achieved via the pump laser. In this case, the OPO signal wavelength remains fixed and the OPO effectively acts as a wavelength converter, transferring the spectral properties of the pump laser (for example linewidth, frequency modulation and tuning range) to the idler output. This approach thus allows the attractive tuning and spectral characteristics available from a variety of near infrared sources to be transferred

Fax: +31 (0)24 36533 11, E-mail: F.harren@science.ru.nl to the mid-infrared. Furthermore, by avoiding direct tuning inputs, the OPO remains mechanically and thermally undisturbed, improving the power and spectral stability of the idler output.

This approach has benefited considerably from the recent availability of fiber-based continuous-wave pump sources, which provide both broad tunability and the watt-level output powers required to efficiently pump a singly-resonant OPO. By contrast, while OPOs pumped by previously available high-power cw pump sources have demonstrated tuning ranges of hundreds of wavenumbers; this could only be achieved by varying the OPO crystal temperature and quasiphase-matching (QPM) period in combination with the highly limited tuning of the pump $[5,6]$. This approach allowed only slow tuning in discrete steps and with limited control accuracy.

In this work we use a fiber-amplified distributed Bragg reflector (DBR) diode laser [7] to pump a continuous wave, singly resonant optical parametric oscillator [6]. Compared to other OPOs with continuous wave pump sources $[8,9]$, this fiber-amplified diode-laser-based source has provided an improved combination of wavelength tuning, simple wavelength setting and long-term active stability. To our knowledge, this OPO has demonstrated the widest mode-hop-free tuning $\left(5.2 \mathrm{~cm}^{-1}\right)$ and the widest $\left(16.5 \mathrm{~cm}^{-1}\right)$ high-resolution, continuous coverage in the mid-infrared by means of changing the pump wavelength. Furthermore, by using a high resolution wavelength meter to provide closed-loop feedback to the pump source, any desired wavelength within the pump tuning range can simply be selected via precise computer control. Thanks to the rapid electronic tuning capability of the DBR laser, the idler wavelength can then be tuned and locked within seconds to an absolute accuracy and stability of $1.7 \times 10^{-3} \mathrm{~cm}^{-1}(1 \sigma)$ determined by the wavelength meter. The idler wavelength was verified to remain locked to the target value for at least $30 \mathrm{~min}$.

The OPO is used in combination with quartz-enhanced photoacoustic spectroscopy (QEPAS) [10] for the detection of trace-level atmospheric components. To date, QEPAS has been used with laser sources such as quantum cascade lasers [11,12] and distributed feedback diode lasers $[10,13,14]$, which both typically have an order of magnitude less power and spectral coverage than an OPO. In common with other photoacoustic methods, and in contrast 
with techniques such as direct absorption, wavelength or frequency modulation spectroscopy [15-17], cavity ring-down spectroscopy $[18,19]$ and cavity enhanced absorption spectroscopy [18], the sensitivity of QEPAS scales with power and can, therefore, directly benefit from the high power of the OPO. In contrast to conventional photoacoustic detection with microphones, QEPAS makes use of a quartz tuning fork (developed as a frequency reference for clocks and watches) as both a detector and high-Q resonator for the acoustic signal. Although the sensitivities that can be reached are comparable to those of conventional photoacoustic spectroscopy, QEPAS has the advantage of a small gas sampling volume $\left(\sim 1 \mathrm{~mm}^{3}\right)$. This volume is ultimately determined by the space between the two prongs of the tuning fork. The acoustic signal is generated by wavelength modulation of the exciting laser at half the resonant frequency of the tuning fork, allowing secondharmonic detection at the resonant frequency leading to strong rejection of acoustic background signals. This necessitates a source capable of wavelength modulation in the kilohertz range. The simplicity with which OPOs of the type described here, pumped by amplified diode lasers, can be modulated at frequencies up to hundreds of $\mathrm{MHz}$ has been recently demonstrated [17] and makes them ideally suited to QEPAS detection. Here we demonstrate the sensitivity and versatility of the OPO combined with this detection method by recording multi-component trace gas mixtures of laboratory air.

\section{2}

\section{Experimental setup}

The experimental setup as shown in Fig. 1 consists of three parts: a fiber-amplified near-infrared DBR diode laser as tunable pump source, a singly resonant OPO that converts the pump wavelength to the mid-infrared region and QEPAS for the sensitive detection and quantification of trace gases.
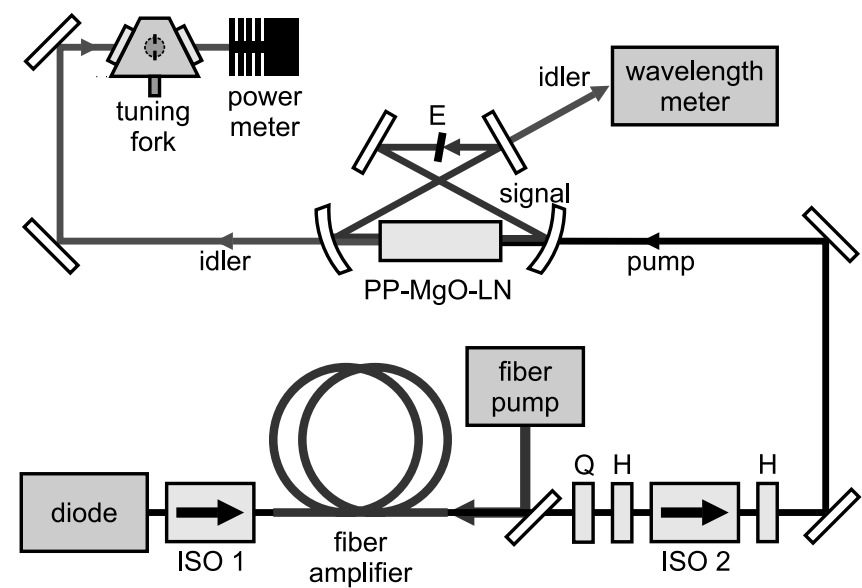

FIGURE 1 Schematic representation of the experimental setup. The out put of a three-section DBR diode laser is seeded, through a $60 \mathrm{~dB}$ optical isolator (ISO 1), into a fiber amplifier, which is pumped by a $25 \mathrm{~W} 976 \mathrm{~nm}$ diode bar. The amplified light passes through a second $30 \mathrm{~dB}$ optical isolator (ISO 2) and quarter- and half-wave plates $(\mathrm{Q}, \mathrm{H})$ for polarization control before pumping a singly resonant optical parametric oscillator based on an $\mathrm{MgO}$-doped periodically poled lithium niobate crystal (PP-MgO-LN). An intra-cavity etalon $(\mathrm{E})$ serves as a frequency selective element for the signal wavelength. A part of the idler is monitored with a wavelength meter, while the majority of the power is used for trace gas detection with quartz-enhanced photoacoustic spectroscopy
The fiber-amplified DBR diode laser is similar to that described previously [7]. The DBR diode laser (Eagleyard Photonics $\mathrm{GmbH}$ ) is a multi-section device providing up to $80 \mathrm{~mW}$ of output power at $1082 \mathrm{~nm}$ with a linewidth of $<40 \mathrm{MHz}$. Currents to the tuning sections of the device were controlled by dedicated scan linearization and synchronization electronics coupled to a computer for automated operation, as indicated in Fig. 2. The diode laser radiation was directed through a two-stage $(60 \mathrm{~dB})$ optical isolator, after which $50 \mathrm{~mW}$ of power remained to seed a $30 \mathrm{~m}$ long double-clad Yb-doped fiber amplifier (Institut für Physikalische Hochtechnologie, Jena, Germany). The amplifier, which had a $10 \mu \mathrm{m}$ core, was end-pumped with up to $25 \mathrm{~W}$ from a fiber-coupled $976 \mathrm{~nm}$ diode laser via a $400 \mu \mathrm{m}$ D-shaped inner cladding. Under certain conditions, the amplifier output exhibited instabilities of a type usually attributed to Brillouin scattering. These were suppressed by applying a $2 \mathrm{MHz}$ sinusoidal modulation to the gain section of the seed laser, thus broadening its spectrum to $\sim 100 \mathrm{MHz}$, as previously described [7]. After passing a second isolator and polarization control, up to $7.9 \mathrm{~W}$ of power at $1082 \mathrm{~nm}$ was available to pump the singly resonant OPO $[6,20]$. Using an AR coated lens $(f=7.5 \mathrm{~cm})$, we focused the pump beam into a $5 \mathrm{~cm}$ long $\mathrm{MgO}$-doped periodically poled lithium niobate crystal (PPMgO-LN) (HC Photonics). This crystal was a multi-grated type with 7 different poling periods ranging from 28.5 to $31.5 \mu \mathrm{m}$. To suppress etalon-effects, one of the two edges had a slight wedge of 1 degree and both sides were anti-reflective coated for the pump, signal and idler wavelengths. An oven was used to keep the crystal at a stable temperature between 50 and $190^{\circ} \mathrm{C}$.

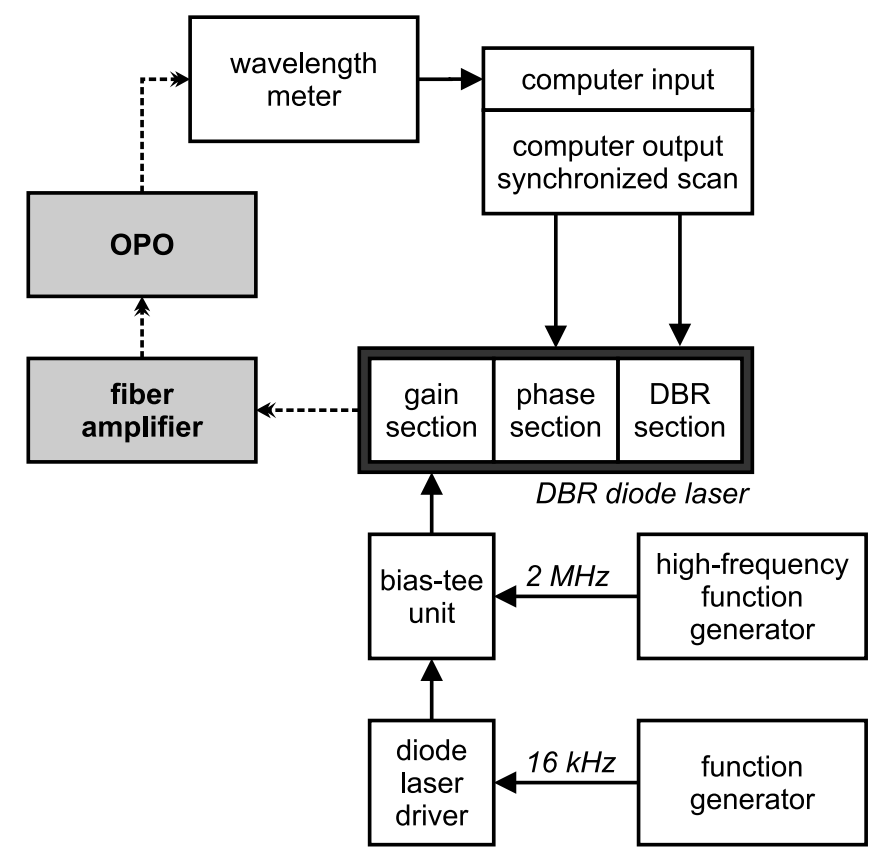

FIGURE 2 Schematic representation of the control and feedback electronics. The three-section DBR diode laser is modulated via the gain section current at $2 \mathrm{MHz}$ to broaden the seed laser bandwidth, as well as at $16.377 \mathrm{kHz}$, which is half the resonance frequency of the tuning fork in QEPAS. A wavelength meter measurement of the idler output is used to drive a computerized closed-loop control system for wavelength tuning and stabilization 
The OPO cavity had a bow-tie configuration and consisted of two meniscus $(R=100 \mathrm{~mm})$ and two plane mirrors which were coated such that they were highly reflective for the signal and transparent for the pump and idler wavelengths. An intracavity solid YAG etalon (uncoated, $0.4 \mathrm{~mm}$ thick) mounted on a galvo-drive was placed at one of the two focal points of the resonated signal beam to serve as a frequency-selective element enhancing stable single-mode operation.

Residual idler, transmitted through one of the plane mirrors, was used for wavelength calibration by a high resolution wavelength meter (Bristol Instruments 621A). The idler wavelength was recorded twice a second and could be determined at an absolute accuracy of $5 \times 10^{-4} \mathrm{~cm}^{-1}$ [21].

The idler output beam was refocused with a set of three AR-coated $\mathrm{ZnSe}$ lenses of focal lengths $100 \mathrm{~mm}, 500 \mathrm{~mm}$ and $75 \mathrm{~mm}$ (ISP optics). The first lens served as a collimating lens, after which two pinholes and two beam steering mirrors followed to redirect the beam through the remaining lenses and the prongs of the tuning fork. The pinholes were used to overlap a He-Ne laser for alignment of the tuning fork. While parasitic up-conversion processes in the OPO also provide a visible output beam, slight wavelength-dependent deviations in optical elements such as the out-coupling meniscus mirror and lenses result in the overlap with the idler being insufficient to use for alignment of the QEPAS cell.

The tuning fork used for these experiments was a quartz crystal resonator (AEL crystals) with a specified frequency of $32.768 \mathrm{kHz}$ in the oscillator circuit, and had a Q-factor of $2.3 \times 10^{4}$ at a $200 \mathrm{mbar}$ pressure. The optical gas cell enclosing the fork had a volume of $3 \mathrm{~cm}^{3} . \mathrm{CaF}_{2}$ windows were installed at Brewster's angle on either end of the cell. The gas intake was positioned just above the tuning fork so that gas would flow directly between the prongs and the effective gas sampling volume was minimized. The gas output was connected to a membrane pump through a needle vent, to regulate the pump capacity, and a dampening volume, to prevent any pump noise from reaching the tuning fork.

For wavelength modulation a sinusoidal signal was applied to the gain section of the DBR laser such that the idler was modulated at $f=16.377 \mathrm{kHz}$, half the resonance frequency of the tuning fork at 200 mbar pressure. The modulation amplitude $\left(4.8 \times 10^{-2} \mathrm{~cm}^{-1}\right)$ was optimized to the width of an ethane absorption line at $2990.1 \mathrm{~cm}^{-1}$. A lock-in amplifier (Stanford Research Systems SRS830) was used to detect the signal generated by the tuning fork in response to the photoacoustic sound wave. The detection was performed at $2 f=32.754 \mathrm{kHz}$ (the resonance frequency of the tuning fork at 200 mbar). To facilitate a clean pass of the idler beam, the acoustic micro-resonators typically used with QEPAS [10] were omitted in this case. Without resonators $81 \%$ of the power remained after the cell. A power meter following the cell allowed calibration of the QEPAS response to the absolute idler power level.

\section{$3 \quad$ OPO tuning and wavelength stabilization}

Tuning of the OPO was accomplished in three steps. The first step was selecting a suitable poling period for coarse tuning of the idler wavelength ( 3 to $4 \mu \mathrm{m}$ within a single PP-MgO-LN crystal). Subsequently, the crystal tempera- ture was adjusted. Though giving a broad tuning capability of hundreds of wavenumbers, discrete steps of $1-4 \mathrm{~cm}^{-1} \mathrm{oc}-$ curred in this process, due to the combination of OPO cavity modes, etalon mode selection and the PP-MgO-LN phasematching bandwidth. In addition, temperature tuning is also a relatively slow method of changing the wavelength.

The third tuning step was pump tuning, which was used for fine wavelength control once coarse wavelength selection had been made by the first two steps. To accomplish this, the phase and DBR sections of the multi-section DBR laser were thermally tuned by passing current through resistive micro-heaters fabricated onto their upper surfaces. Synchronous tuning of the phase and DBR sections allowed for mode-hop-free tuning over $5.2 \mathrm{~cm}^{-1}$ at the idler wavelength, as shown in Fig. 3, while varying the DBR section offset current allowed this mode-hop-free range to be shifted within a total range of $16.5 \mathrm{~cm}^{-1}$ as demonstrated in Fig. 4. The complete scan shown in Fig. 3 was performed in 2 min, this was mainly limited by the $2 \mathrm{~Hz}$ sampling rate of the wavelength

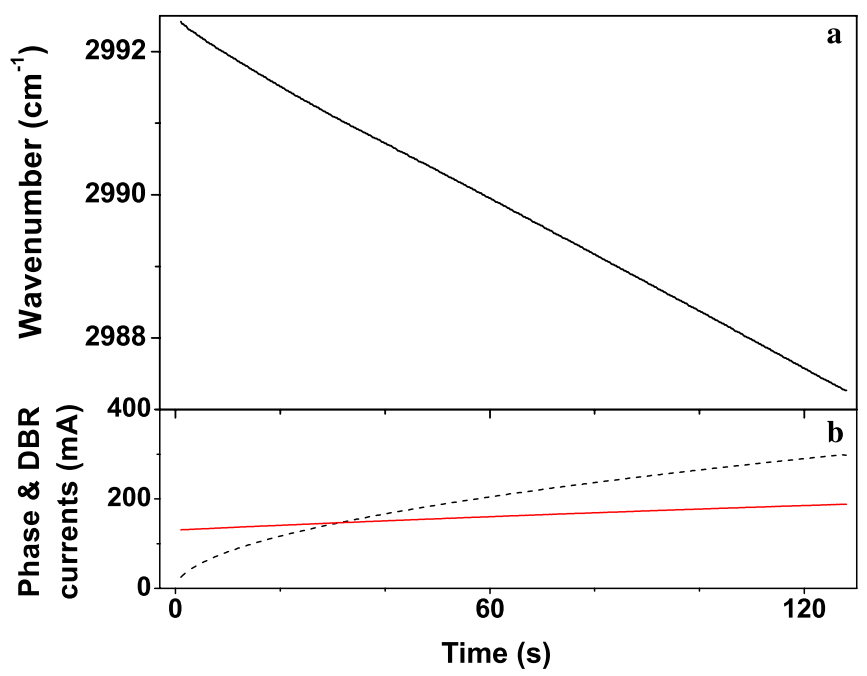

FIGURE 3 Mode-hop-free scan over $5.15 \mathrm{~cm}^{-1}$ in the idler wavelength by synchronized tuning of the phase (b, dashed line) and DBR section (b, solid line) of the seed diode laser. The tuning rate in this measurement was limited by the wavelength meter, which had a sampling frequency of $2 \mathrm{~Hz}$

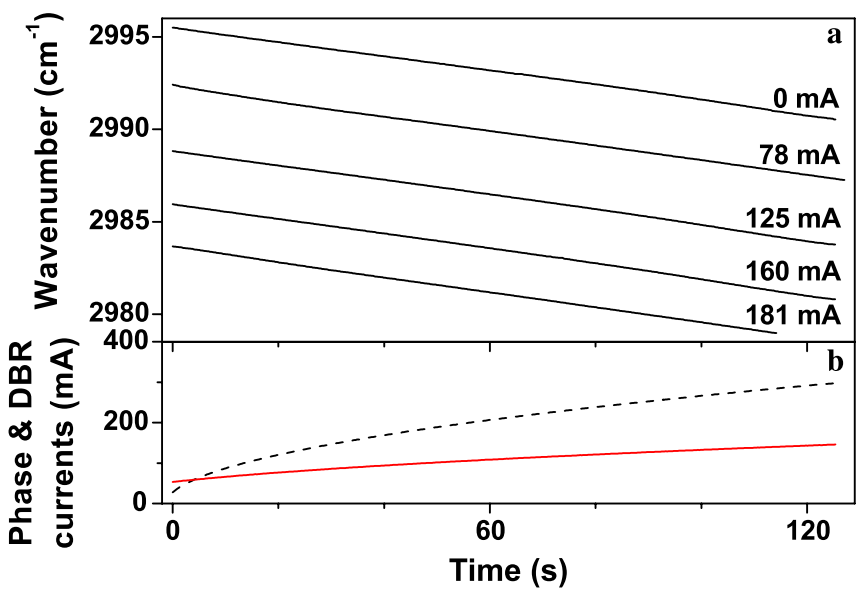

FIGURE 4 (a) Continuous, high-resolution scan over $16.5 \mathrm{~cm}^{-1}$ was achieved by performing synchronized phase/DBR scans at different DBR offset currents (indicated in $\mathrm{mA}$ ); (b) DBR and phase-section current variation corresponding to the scan with DBR offset of $0 \mathrm{~mA}$ 
meter and the number of recorded points. Previously, we have demonstrated that such a continuous scan can be achieved on millisecond timescales [7].

The rapid tuning capability of the DBR laser combined with online monitoring of the idler wavelength by the wavelength meter allowed implementation of a simple feedback system to set and lock the desired idler wavelength within the tuning range of the pump source. The wavelength meter sent the absolute idler wavelength to a computer system, which in turn adjusted the phase and DBR currents as required. Figure 5a demonstrates locking of the idler at $2990.076 \mathrm{~cm}^{-1}$ showing a stability of $1.7 \times 10^{-3} \mathrm{~cm}^{-1}(1 \sigma)$ over $30 \mathrm{~min}$. The currents sent to the phase and DBR section to maintain this stability are indicated in Fig. 5b. It can be seen that, apart from fast corrections, a slower adjustment to the DBR laser currents was required which can be attributed to the compensation of slow variations in environmental conditions. It should be noted that no active stabilization of the OPO signal wavelength was attempted.

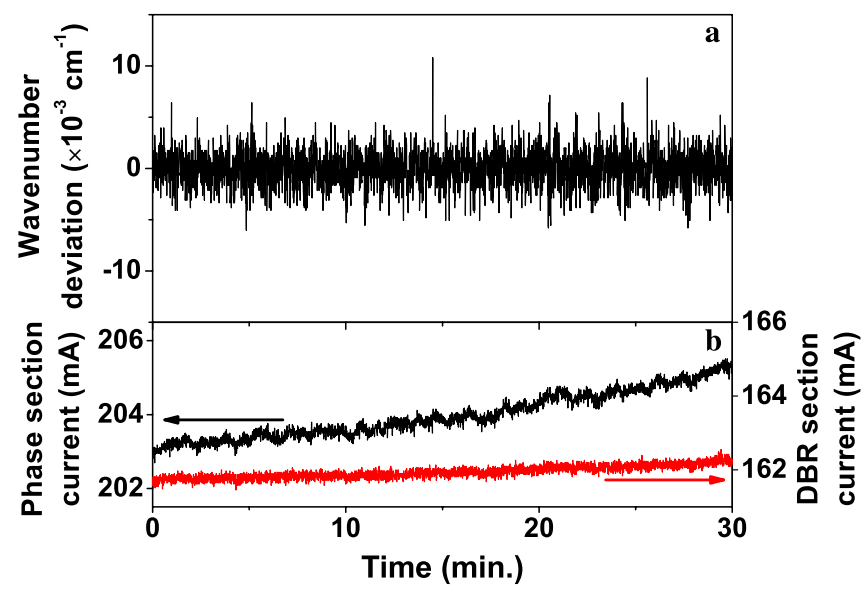

FIGURE 5 (a) Locked idler wavelength showing a stability of $1.7 \times 10^{-3} \mathrm{~cm}^{-1}$ over $30 \mathrm{~min}$. The vertical axis shows the deviation from the set wavelength at $2990.076 \mathrm{~cm}^{-1}$. (b) The currents to the phase and DBR sections of the diode laser were adjusted continuously by the feedback system

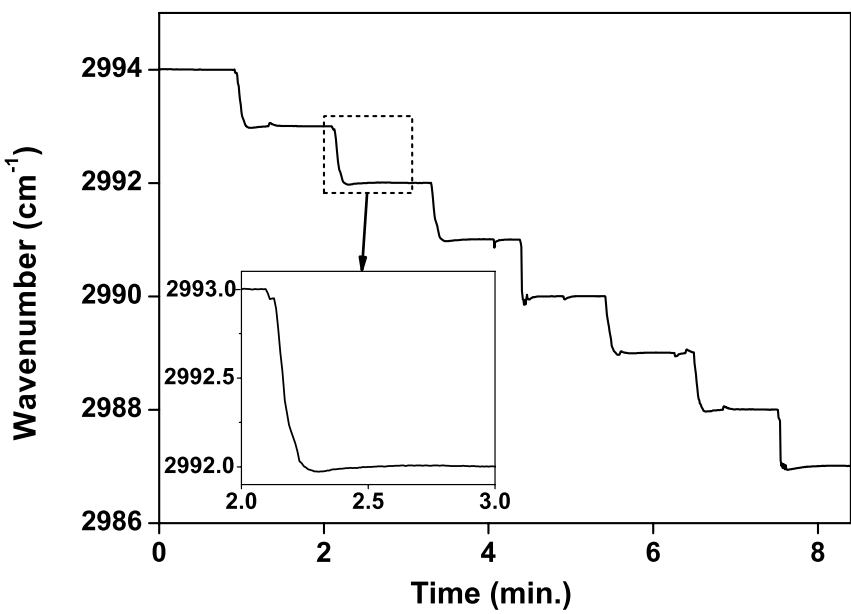

FIGURE 6 Setting and locking of the idler wavelength by pump wavelength tuning is demonstrated for the range $2994 \mathrm{~cm}^{-1}$ to $2987 \mathrm{~cm}^{-1}$ with steps of $1 \mathrm{~cm}^{-1}$. After setting a new wavelength, the lock is acquired in $\sim 20 \mathrm{~s}$ (inset)
The computer controlled wavelength read-out and feedback system to the DBR laser makes it simple to select any wavelength within the pump tuning range. In Fig. 6 this is demonstrated for a set of wavenumbers from 2987 to $2994 \mathrm{~cm}^{-1}$ with programmed tuning in $1 \mathrm{~cm}^{-1}$ steps. The inset shows that the desired wavelength is reached within $\sim 20 \mathrm{~s}$, limited by the wavelength meter and locking algorithm, and then locked for a 1 min time span. Occasionally a mode hop could occur within the OPO especially after the wavelength was changed, but this was swiftly corrected by the feedback system via the DBR laser tuning controls. This flexible control system clearly has the potential to rapidly tune on and off resonance for a specific absorption line or to rapidly switch between absorption features of different species.

\section{QEPAS measurements of ethane}

Figure 7 shows an example of QEPAS spectral data recorded by scanning over an absorption feature at $2990.1 \mathrm{~cm}^{-1}$ in 2 ppmv (parts-per-million by volume) ethane, buffered at 200 mbar in pure nitrogen, with a wavelength modulation amplitude of $4.8 \times 10^{-2} \mathrm{~cm}^{-1}$. The continuous scan, consisting of 500 data points (300 ms per point), was recorded in $150 \mathrm{~s}$ with a lock-in time constant of $1 \mathrm{~s}(24 \mathrm{~dB} /$ oct filter slope, $\Delta f=0.156 \mathrm{~Hz}$ ). The signal was thus smoothed by the lock-in amplifier. The measured ethane absorption feature is built up of multiple lines, giving rise to a broadened, asymmetric version of the pure second derivative signal usually obtained from an isolated absorption feature in QEPAS. In the same figure, the filled circles represents a background measurement in nitrogen, which shows that observed structure in the ethane measurement is genuinely due to absorption rather than fringing or other noise sources. Most of these minor absorption features are not listed in the HITRAN database. In particular, HITRAN-based modeling of the $2 f$-signal yields a single peak, not a double peak structure revealed in Fig. 7. In the inset of Fig. 7 a QEPAS

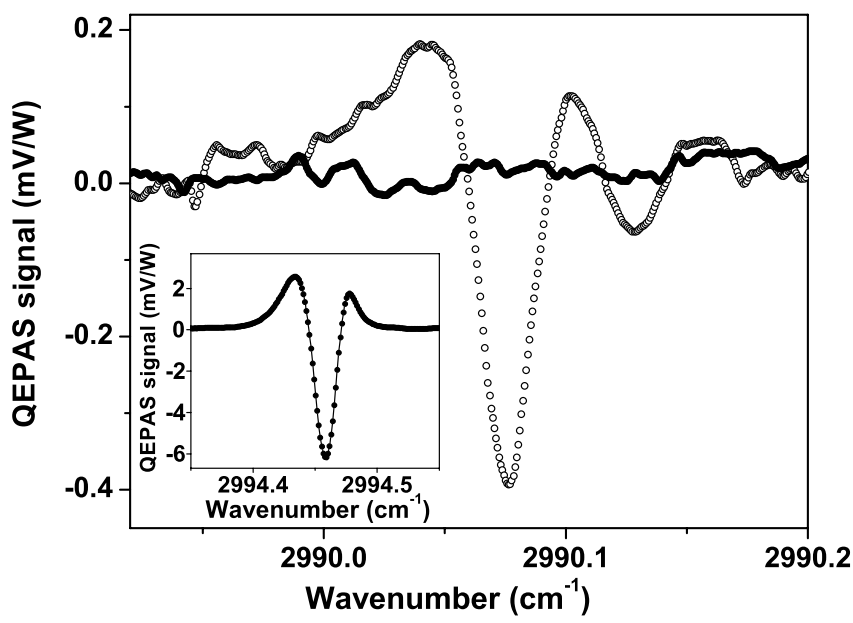

FIGURE 7 Example of a QEPAS scan over a 2 ppmv ethane peak at $2990.08 \mathrm{~cm}^{-1}$ (open circles) in 200 mbar nitrogen gas. QEPAS signal detected from pure nitrogen in the same spectral range is also shown, revealing a flat background signal (filled circles). Another example of a QEPAS scan is given in the inset for $1.2 \%$ water at $2994.4 \mathrm{~cm}^{-1}$ 


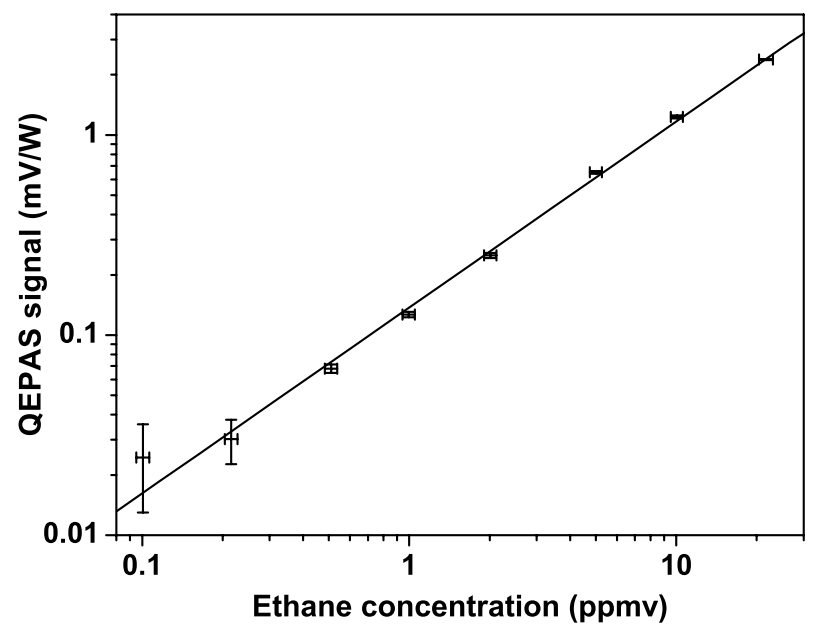

FIGURE 8 Ethane peak at $2990.08 \mathrm{~cm}^{-1}$ measured at different concentrations showing the linear response of QEPAS. Each point in the graph represents 100 measurements from which a standard deviation could be determined. This resulted in a detection limit of 25 ppbv over $20 \mathrm{~s}$

recording of an isolated $1.2 \%$ water absorption peak at $2994.4 \mathrm{~cm}^{-1}$ is given, showing the expected second derivative form.

The sensitivity and linearity of the OPO-QEPAS system were demonstrated by locking the laser at the maximum of the absorption peak, measuring concentrations ranging from 20 ppmv down to $100 \mathrm{ppbv}$ and performing a linear fit (Fig. 8). Each concentration point in the graph represents 100 data points from which the standard deviation was determined. This resulted in a detection limit of $25 \mathrm{ppbv}$ (parts-per-billion by volume) with a lock-in time constant of $3 \mathrm{~s}(12 \mathrm{~dB} /$ oct filter slope, $\Delta f=0.083 \mathrm{~Hz}$ ), an averaging time of $20 \mathrm{~s}$ and a laser power of $217 \mathrm{~mW}$ measured behind the cell. The corresponding normalized noise equivalent absorption coefficient $(1 \sigma)$ is $7.0 \times 10^{-7} \mathrm{~cm}^{-1} \mathrm{~W} / \mathrm{Hz}^{1 / 2}$. Compared to previous results [10] this is about one order of magnitude less sensitive. The main reason for this is the lack of microresonator enhancement of the QEPAS signal.

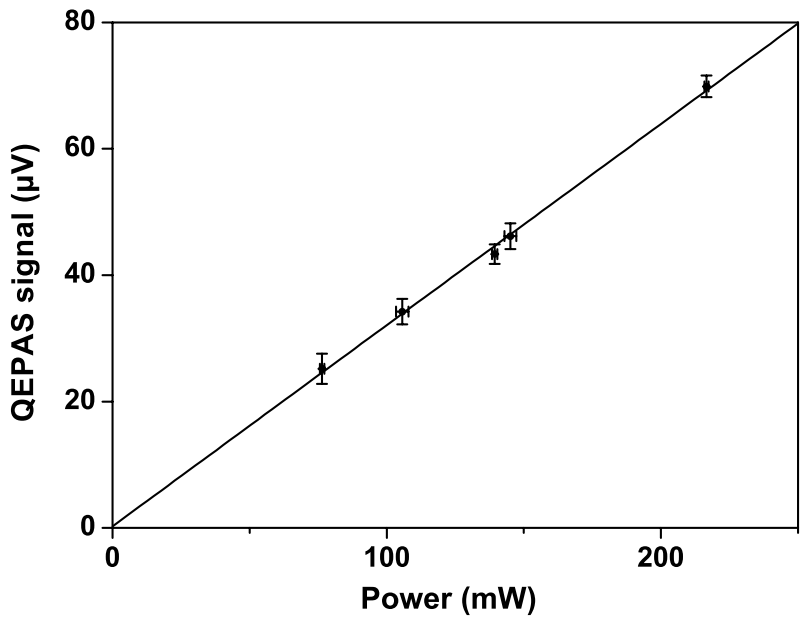

FIGURE 9 Power dependence of the QEPAS signal by locking the idler wavelength to the maximum of the ethane absorption peak at $2990.08 \mathrm{~cm}^{-1}$ and varying the fiber amplifier pump power or attenuating the OPO idler beam. A linear response to the power was found, proving the advantage of higher powers for QEPAS

5

\section{Power dependence of the QEPAS response}

These experiments are the first in which such a high power is used with QEPAS. Therefore the power-dependence of the QEPAS signal was measured by keeping the idler wavelength fixed at the same ethane absorption peak with a concentration of 2 ppmv (Fig. 9). The graph clearly shows the expected linear response of the QEPAS signal. The power of the OPO was varied either by reducing the pump power to the fiber amplifier or by attenuating the output beam of the OPO.

6

\section{Spectral scan of a multi-component gas mixture of laboratory air and ethane over $15 \mathrm{~cm}^{-1}$}

A key advantage of using an OPO for trace-gas a spectroscopy lies in its ability to cover wide spectral ranges and thereby detect multiple gas species. This was demonstrated by pump-tuning the OPO, as described above, from $2979.4 \mathrm{~cm}^{-1}$ to $2994.6 \mathrm{~cm}^{-1}$ while recording a QEPAS spec-

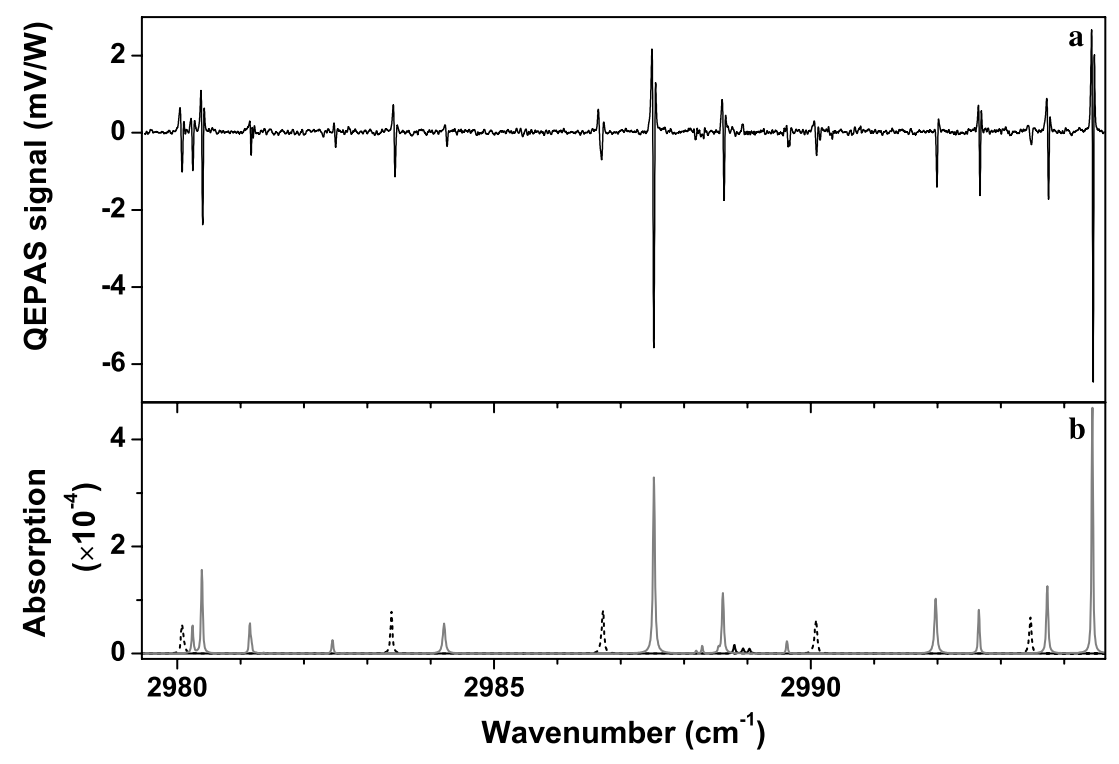

FIGURE 10 (a) QEPAS recording of a laboratory air and an ethane/nitrogen mixture, demonstrating the multi-component detection capability over a broad spectral range of $15.1 \mathrm{~cm}^{-1}$. (b) Calculated HITRAN data for $2.2 \mathrm{ppmv}$ ethane (dashed line), $1.53 \mathrm{ppmv}$ methane (black line) and $1.1 \%$ water (gray line) at $200 \mathrm{mbar}$ 
trum (Fig. 10a). The time in which the spectrum was recorded was $11 / 2 \mathrm{~h}$ and the lock-in time constant was $1 \mathrm{~s}$. The complete spectral scan consists of 13000 points at $\sim 400 \mathrm{~ms}$ per point. The 200 mbar gas mixture was created by mixing a certified gas mixture of ethane with laboratory air in a $1: 9$ ratio, thus obtaining $2.2 \mathrm{ppmv}$ ethane, $1.1 \%$ water (measured with a hygrometer) and $1.5 \mathrm{ppmv}$ methane (estimated from typical air concentration). The scan was performed faster than the inverse lock-in amplifier bandwidth would require, which explains a noticeable asymmetric line shape. However, a comparison with a calculated spectrum based on HITRAN data [22] shows good agreement for the absorptions of all three compounds (Fig. 10b). For the calculations a pseudoVoigt profile was used as described by Liu et al. [23].

From the recorded spectrum it can be seen that by selecting the ethane peak at $2983.3 \mathrm{~cm}^{-1}$ instead of the initial peak at $2990.1 \mathrm{~cm}^{-1}$, the detection limit can be improved from $25 \mathrm{ppbv}$ to $13 \mathrm{ppbv}$. This corresponds to a normalized noise equivalent absorption of $4.4 \times 10^{-7} \mathrm{~cm}^{-1} \mathrm{~W} / \mathrm{Hz}^{1 / 2}$. In a similar fashion, the detection limit for the water peak at $2994.4 \mathrm{~cm}^{-1}$ can be estimated to be $6.8 \mathrm{ppmv}$ for a data acquisition time of $20 \mathrm{~s}$.

\section{Conclusion}

In summary, we have reported on a continuous wave, singly resonant OPO for the 3 to $4 \mu \mathrm{m}$ spectral range with an average output power of $300 \mathrm{~mW}$. The OPO was pumped by a fiber-amplified DBR laser which gave significant advantages in terms of wavelength tunability and longterm stability. Online monitoring of the idler wavelength was used to provide closed-loop tuning control of the OPO by tuning the DBR laser, making it possible to simply set any wavelength within the pump tuning range and lock it to an absolute accuracy of $1.7 \times 10^{-3} \mathrm{~cm}^{-1}(1 \sigma)$ over $30 \mathrm{~min}$. In addition, mode-hop-free tuning (over $5.2 \mathrm{~cm}^{-1}$ ) and tuning with continuous coverage (over $16.5 \mathrm{~cm}^{-1}$ ) was demonstrated by synchronized tuning of the phase and DBR sections of the seeding diode laser. The combination of high power in the mid-infrared, wide tunability, programmed wavelength access and simplicity of modulation at $\mathrm{kHz}$ frequencies makes the OPO system a highly attractive source for rapid trace-level detection of multiple gas species by QEPAS.

The small dimensions of the QEPAS cell make it an ideal detection method for building compact gas detectors if combined with smaller light sources, such as diode lasers or quantum cascade lasers. In a laboratory environment however, an OPO with QEPAS offers benefits that would not be available with other sources, these being a high output power and broad spectral coverage. Furthermore, trace gas detection can be performed within a small gas cell volume so that minute gas samples can be analyzed and dynamic changes in concentration can be followed. The linear dependence of the QEPAS signal on the power demonstrates both the benefits of using higher power lasers with QEPAS and the potential for further sensitivity gains by power scaling to the watt-level outputs achievable with such OPOs [5-7,24]. From a long, multicomponent scan, detection limits were obtained of 13 ppbv for ethane and 6.8 ppmv for water (20 s averaging). These values correspond to a normalized noise equivalent absorption of $4.4 \times 10^{-7} \mathrm{~cm}^{-1} \mathrm{~W} / \mathrm{Hz}^{1 / 2}$.

ACKNOWLEDGEMENTS This research was financially supported by the Dutch Technologiestichting STW and the European Commission under the program New and Emerging Science and Technologies, contract number FP6-NESTA-0025042 'Optical Nose'. I.D. Lindsay acknowledges support of the European Commission in the form of a Marie Curie Intra-European Fellowship.

\section{REFERENCES}

1 P.E. Powers, T.J. Kulp, S.E. Bisson, Opt. Lett. 23, 159 (1998)

2 S.E. Bisson, K.M. Armstrong, T.J. Kulp, M. Hartings, Appl. Opt. 40, 6049 (2001)

3 M.M.J.W. van Herpen, S.E. Bisson, F.J.M. Harren, Opt. Lett. 28, 2497 (2003)

4 F. Müller, A. Popp, F. Kühnemann, S. Schiller, Opt. Express 11, 2820 (2003)

5 M.M.J.W. van Herpen, S.E. Bisson, A.K.Y. Ngai, F.J.M. Harren, Appl. Phys. B 78, 281 (2004)

6 A.K.Y. Ngai, S.T. Persijn, G. von Basum, F.J.M. Harren, Appl. Phys. B 85, 173 (2006)

7 I.D. Lindsay, B. Adhimoolam, P. Gross, M.E. Klein, K.J. Boller, Opt. Express 13, 1234 (2005)

8 A. Henderson, R. Stafford, Opt. Express 14, 767 (2006)

9 F. Müller, G. von Basum, A. Popp, D. Halmer, P. Hering, M. Mürtz, F. Kühnemann, S. Schiller, Appl. Phys. B 80, 307 (2005)

10 A.A. Kosterev, F.K. Tittel, D.V. Serebryakov, A.L. Malinovsky, I.V. Morozov, Rev. Sci. Instrum. 76, 043105 (2005)

11 A.A. Kosterev, Y.A. Bakhirkin, F.K. Tittel, Appl. Phys. B 80, 133 (2005)

12 M.D. Wojcik, M.C. Phillips, B.D. Cannon, M.S. Taubman, Appl. Phys. B 85, 307 (2006)

13 G. Wysocki, A.A. Kosterev, F.K. Tittel, Appl. Phys. B 85, 301 (2006)

14 R. Lewicki, G. Wysocki, A.A. Kosterev, F.K. Tittel, Appl. Phys. B 87, 157 (2007)

15 J.A. Silver, Appl. Opt. 31, 707 (1992)

16 F.K. Tittel, D. Richter, A. Fried, Mid-Infrared Laser Applications in Spectroscopy, In: Solid-State Mid-Infrared Laser Sources (Springer, Berlin, 2003)

17 I.D. Lindsay, P. Gross, C.J. Lee, B. Adhimoolam, K.J. Boller, Opt. Express 14, 12341 (2006)

18 G. Berden, R. Peeters, G. Meijer, Int. Rev. Phys. Chem. 19, 565 (2000)

19 G. von Basum, D. Halmer, P. Hering, M. Mürtz, S. Schiller, F. Müller, A. Popp, F. Kühnemann, Opt. Lett. 29, 797 (2004)

20 A.K.Y. Ngai, S.T. Persijn, F.J.M. Harren, H. Verbraak, H. Linnartz, Appl. Phys. Lett. 90, 081109 (2007)

21 H. Verbraak, A.K.Y. Ngai, S.T. Persijn, F.J.M. Harren, H. Linnartz, Chem. Phys. Lett. 442, 145 (2007)

22 L.S. Rothman, D. Jacquemart, A. Barbe, D.C. Benner, M. Birk, L.R. Brown, M.R. Carleer, C. Chackerian, K. Chance, L.H. Coudert, V. Dana, V.M. Devi, J.M. Flaud, R.R. Gamache, A. Goldman, J.M. Hartmann, K.W. Jucks, A.G. Maki, J.Y. Mandin, S.T. Massie, J. Orphal, A. Perrin, C.P. Rinsland, M.A.H. Smith, J. Tennyson, R.N. Tolchenov, R.A. Toth, J. Vander Auwera, P. Varanasi, G. Wagner, J. Quant. Spectrosc. Radiat. Transf. 96, 139 (2005)

23 Y.Y. Liu, J.L. Lin, G.M. Huang, Y.Q. Guo, C.X. Duan, J. Opt. Soc. Am. B 18, 666 (2001)

24 M.E. Klein, P. Gross, K.J. Boller, M. Auerbach, P. Wessels, C. Fallnich, Opt. Lett. 28, 920 (2003) 